\title{
Surgical repair of a proximal patellar tendon avulsion in a dog utilizing triple patellar bone tunnels and modified tendon repair technique
}

\author{
Matthew D. Johnson ${ }^{1, *}$, Diego Rafael Sobrino ${ }^{1,2}$, Daniel Dean Lewis ${ }^{1}$ and Justin Shmalberg ${ }^{1,3}$ \\ ${ }^{l}$ Department of Small Animal Clinical Sciences, College of Veterinary Medicine, University of Florida, Gainesville, \\ FL 32610-0126, USA \\ ${ }^{2}$ Current Address: Aloha Pet and Bird Hospital, 968 E. Eau Gallie Blvd., Indian Harbour Beach, FL 32937, USA \\ ${ }^{3}$ Current Address: Department of Comparative, Diagnostic \& Population Medicine, College of Veterinary Medicine, \\ University of Florida, Gainesville, FL 32610-0126 USA
}

\begin{abstract}
A three-and-a-half-year-old neutered male Standard Schnauzer presented with a 5-week history of inability to bear full weight on the right pelvic limb following a traumatic injury to the right stifle. Clinical, radiographic, and ultrasound findings were consistent with a proximal patellar tendon rupture with a distal patellar avulsion fragment. A proprietary Achilles repair jig system was used to reattach the patellar tendon to the patella, allowing for direct approximation of the tissues. The primary repair was supported with a protective patella-to-tibia suture, and the dog was fitted with an orthosis to restrict range of motion of the stifle. Primary tendon repair using alternative suture materials and suture placement, in conjunction with a protective suture loop and transient stifle immobilization, allowed for a successful outcome without appreciable lameness at a final evaluation 3.6 years after presentation. The dog in this report was successfully treated with a modified proprietary Achilles' tendon repair system using previously undescribed paired axial patellar bone tunnels in conjunction with a patello-tibial protective ultrahigh molecular weight polyethylene suture. This technique allowed apposition of the tendon to the bone and a stable tenorrhaphy to the patella.
\end{abstract}

Keywords: Patellar Tendon, Tenorrhaphy.

\section{Introduction}

Patellar tendon rupture is an uncommon injury in dogs (Shipov et al., 2008; Das et al., 2014). The patellar tendon is the terminal insertion of the quadriceps mechanism and contributes to stifle extension (Hermanson, 2012). Disruption of the patellar tendon prevents active stifle extension and causes involuntary stifle collapse during the stance phase (Kowaleski et al., 2012). Reported causes of patellar tendon rupture in dogs include direct or indirect trauma (Smith et al., 2000; Shipov et al., 2008), steroid-associated degeneration (Smith et al., 2000), or spontaneous rupture after fluoroquinolone administration (Cabassu et al., 2001).

Direct patellar tendon trauma can be caused by a laceration or a blunt injury to the stifle resulting in complete or partial tendon disruption (Smith et al., 2000). Rupture of the patellar tendon by indirect trauma occurs with quadriceps contraction during forced stifle flexion (Shipov et al., 2008). A number of techniques have been described to address patellar tendon insufficiency in dogs; most advocate ancillary stabilization to protect the primary repair (Smith et al., 2000; Cabassu et al., 2001; Shipov et al., 2008; Das et al., 2014). Previously reported techniques describe primary tenorrhaphy, biomechanically supported with an adjunctive circumpatellar or transpatellar-to-tibia nylon or stainless steel prosthesis that functions as an internal splint (Smith et al., 2000; Cabassu et al., 2001; Shipov et al., 2008; Das et al., 2014). Tertiary level protection is provided by temporary immobilization of the stifle with either external coaptation or a transarticular external skeletal fixator (taESF) (Shipov et al., 2008; Das et al., 2014).

Successful tenorrhaphy is dependent on having sufficient tendon parenchyma proximal and distal to the ruptured tendon to place sutures. Several suture patterns are described for the primary repair of tendons and ligaments (Moores et al., 2004; Montgomery and Fitch, 2015; DeCamp et al., 2016). Ruptures at the tendon's origin or insertion require the use of bone tunnels or anchors that allow the tendon to be reattached to bone (Moores et al., 2004). The injury described in this case did not have sufficient tendon parenchyma available for primary tenorrhaphy and the application of a modified three loop pulley technique would likely damage the articular cartilage of the femoral trochlea.

The purpose of this report is to describe a new technique for proximal patellar tendon avulsion repair 
utilizing a proprietary Achilles' tendon repair system and previously undescribed axial patellar bone tunnels in conjunction with a patellotibial protective ultrahigh molecular weight polyethylene suture.

\section{Case Details}

\section{History}

A 3.5-year-old, male neutered Standard Schnauzer weighing $22 \mathrm{~kg}$ presented with a 5-week history of right pelvic limb lameness ascribed to an injury the dog sustained leaping from the top of a staircase. The dog landed with the right stifle making direct impact with the stairs approximately 5 meters below. The dog sustained a full thickness skin wound over the cranial aspect of the right stifle. The dog was presented to a local emergency facility for evaluation where the dog was sedated for radiographs and wound care. No osseous abnormalities were identified on radiographs. The wound was cleaned and primarily closed. The dog received dexamethasone $(0.09 \mathrm{mg} / \mathrm{kg} \mathrm{SQ})$ (DexaJect, Butler Schein Animal Health, Dublin, OH, USA) and ampicillin (12 mg/kg SQ) (Polyflex, Boehringer Ingelheim Vetmedica, Inc. St. Joseph, MO, USA) injections and discharged with oral cephalexin (21 $\mathrm{mg} / \mathrm{kg}$ PO every 12 hours) (Karalex Pharma, LLC, Princeton, NJ, USA) and carprofen ( $2 \mathrm{mg} / \mathrm{kg}$ PO every 12 hours) (Rimadyl, Pfizer Animal Health, New York, NY, USA) for 7 days. The dog was unable to support weight on the right hind limb when it returned to the emergency clinic 11 days later. Fourteen additional days of carprofen were dispensed and the owners were instructed to monitor the dog for improvement.

\section{Clinical examination}

The dog was referred to the University of Florida Small Animal Hospital 45 days after sustaining its injury. The dog attempted to bear weight on the right pelvic limb while standing, and placed nominal weight on the right pelvic limb while walking. The right stifle collapsed involuntarily during stance phase, and remained flexed through the remainder of the gait cycle. Normal voluntary stifle extension was not observed. Diffuse, severe thickening was palpated on the medial, cranial and lateral aspects of the right stifle.

The patella was difficult to palpate, but was proximally displaced and did not change position when the stifle was flexed and extended. Mild crepitus was identified during flexion and extension of the right stifle without overt pain. Cranial drawer and cranial tibial thrust were absent. The remainder of the physical, orthopedic, and neurologic examinations were unremarkable.

\section{Diagnostic imaging}

Flexed lateral and extended craniocaudal radiographic projections of the right stifle were obtained (Figure 1). The patella was located proximally in the trochlear groove and the pre-operative patellar tendon length to patella length ratio (PTL: PL) was measured as 2.97 (Mostafa et al., 2008).

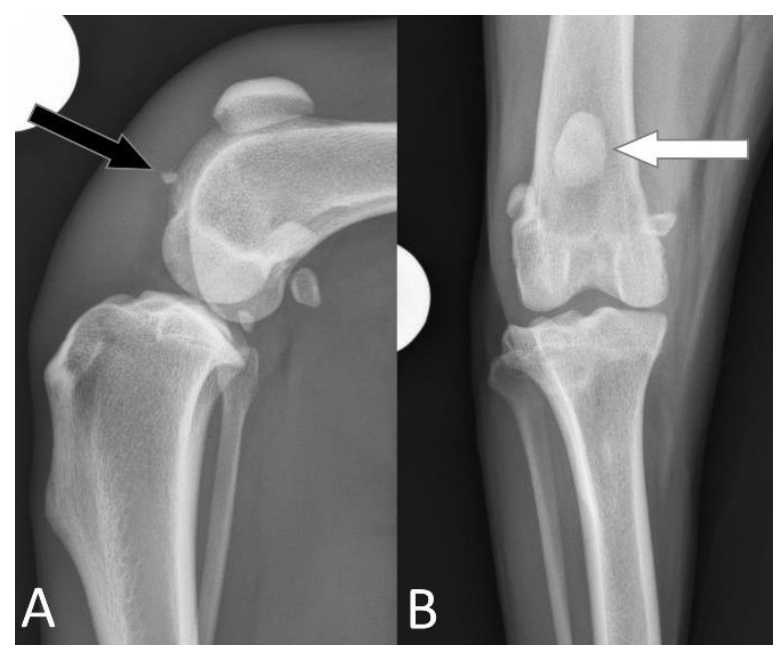

Fig. 1. (A): Lateral projection radiograph of the flexed stifle reveals severe soft tissue opacity in the cranial stifle region. A round, well-defined mineral opacity is found distal to the patella and cranial to the femoral condyles, consistent with an avulsion fracture of the distal pole of the patella (black arrow). (B): Craniocaudal projection of the extended stifle reveals the patella is proximally displaced suggestive of a patellar tendon rupture (white arrow).

The region of the patellar tendon displayed increased soft tissue opacity with a maximal cranial-to-caudal measurement of approximately $15 \mathrm{~mm}$. There was an approximately $3.4 \mathrm{~mm}$ round, well-defined mineral opaque body with smooth margins that was located approximately $1 \mathrm{~cm}$ distal to the patella and craniodistal to the femoral trochlear ridges.

A diagnostic ultrasonic evaluation of the affected stifle was performed. A focal area of fiber disruption and severe thickening of the proximal patellar tendon was identified, and the patellar tendon thickness abruptly decreased to a width of $1 \mathrm{~mm}$ proximally (Figure 2 ). Continuous organized tendon striations were not identified in the proximal patellar tendon adjacent to the apex of the patella. An approximately $3 \mathrm{~mm}$ hyperechoic structure with distal acoustic shadowing was identified distal to the patella and the femoral trochlear groove. The combined radiographic and ultrasound studies were most suggestive of a proximal patellar tendon rupture, with an avulsion fragment from the distal patella, and secondary fibrosis.

\section{Surgical treatment}

The dog was pre-medicated with methadone $(0.3$ $\mathrm{mg} / \mathrm{kg}$, IM) (Mylan Institutional LLC, Rockford, IL, USA) and dexmedetomidine (5 $\mu \mathrm{g} / \mathrm{kg}$, IM) (Zoetis, Kalamazoo, MI, USA). Anesthesia was induced with propofol $(2.3 \mathrm{mg} / \mathrm{kg}$, IV) (Abbott Laboratories, North Chicago, IL, USA) and midazolam (2.3 mg/kg, IV) (West-Ward Phamaceuticals, Eatontown, NJ, USA). The dog was intubated and maintained under general anesthesia with an isoflurane (Piramal Healthcare Limited, Andhra Pradesh, India) $/ \mathrm{O}_{2}$ mixture. 


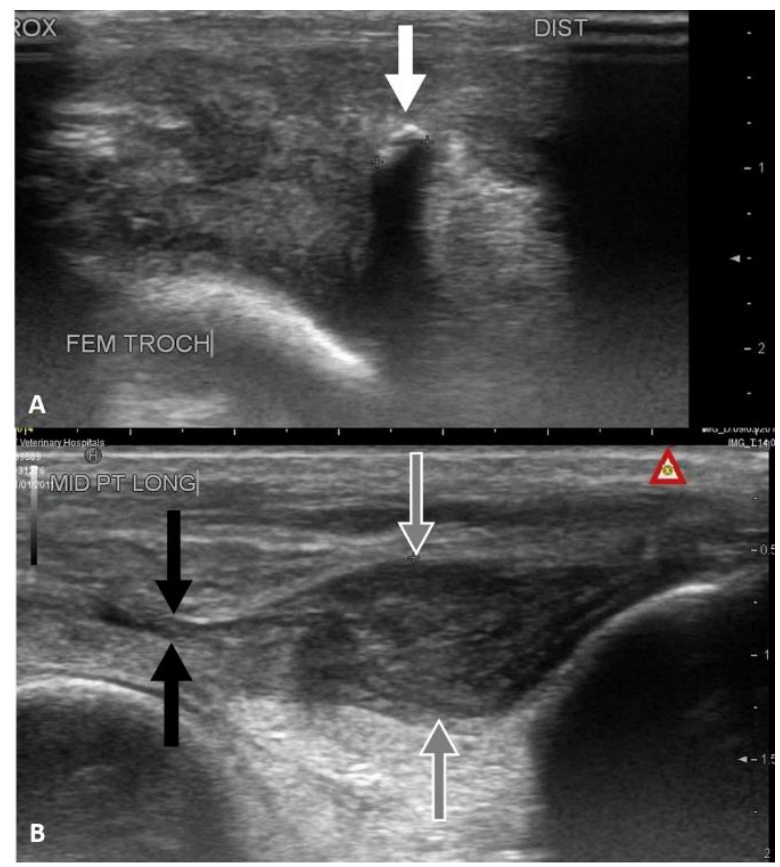

Fig. 2. Longitudinal ultrasonographic image of the cranial stifle in the region of the normal patellar tendon. (A): A hyperechoic structure distal to the femoral trochlear groove with distal acoustic shadowing is present (white arrow). (B): The patellar tendon has a focal area of fiber disruption and severe thickening (gray arrows). The patellar tendon abruptly tapers (black arrows) and cannot be traced proximally.

Antibiotics were administered (cefazolin, $22 \mathrm{mg} / \mathrm{kg}$, IV (Hospira Inc, Lake Forest, IL, USA)), pre-operatively, every 90 minutes intraoperatively, and every 6 hours postoperatively until the following morning. The dog was placed on a constant rate infusion of hydromorphone $(0.02 \mathrm{mg} / \mathrm{kg} / \mathrm{hr}$, IV) (West-Ward Phamaceuticals, Eatontown, NJ, USA), lidocaine (3 $\mathrm{mg} / \mathrm{kg} / \mathrm{hr}$, IV) (MWI, Boise, ID, USA), and ketamine $(0.6 \mathrm{mg} / \mathrm{kg} / \mathrm{hr}$, IV) (Putney Inc, Portland, ME, USA) to maintain analgesia during the procedure.

A right stifle arthrotomy was performed using a craniomedial parapatellar approach. (Johnson, 2014) Normal patellar tendon tissue could not be identified immediately distal to the proximally displaced patella. The patellar fragment was identified cranial to the intercondylar notch within the avulsed patellar tendon. Dense fibrous tissue surrounded the patellar tendon, and dissection of the tendon required debridement of the fibrous tissue between the avulsion fragment and the base of the patella.

The avulsed fragment was too small for use in the repair and was left within the tendon substance. Excised fibrous tissue was submitted for histopathological analysis, that later revealed normal collagen deposition in response to tendon injury without evidence of infection.
A proprietary Achilles tendon repair jig system (PARS, Arthex Inc, Naples, FL USA) was utilized to perform the tenorrhaphy. The PARS system consisted of a custom jig, six strands of \#2 FiberWire and two specialty looped end sutures (Arthrex Inc, Naples, FL USA). The jig is designed for precise placement of three FiberWire suture strands within each portion of a ruptured tendon via six guide holes. The PARS system was used in a modified manner to perform the primary patellar tendon repair. Only three of the six strand of FiberWire were utilized in the dog reported here because the tendon was avulsed. The FiberWire sutures were soaked in a cefazolin solution $(100 \mathrm{mg} / \mathrm{ml})$ prior to implantation. Each strand of suture had a straight needle swedged on the terminal end of the suture to facilitate passage through the jig and tendon. The proximal and distal strands were placed transversely through the tendon. The middle strand was passed first transversely through the tendon followed by removal of the swedged on needle. The looped end specialty sutures were then passed obliquely proximal to the middle strand of suture but distal to the proximal transverse suture. The free ends of the middle suture strand were then passed around, then threaded through the looped end of the oblique sutures. The looped end sutures were pulled through the tendon bringing the terminal ends of the middle suture strand back through the tendon for a second pass forming a self-locking loop on each abaxial aspect of the tendon (Figure 3).

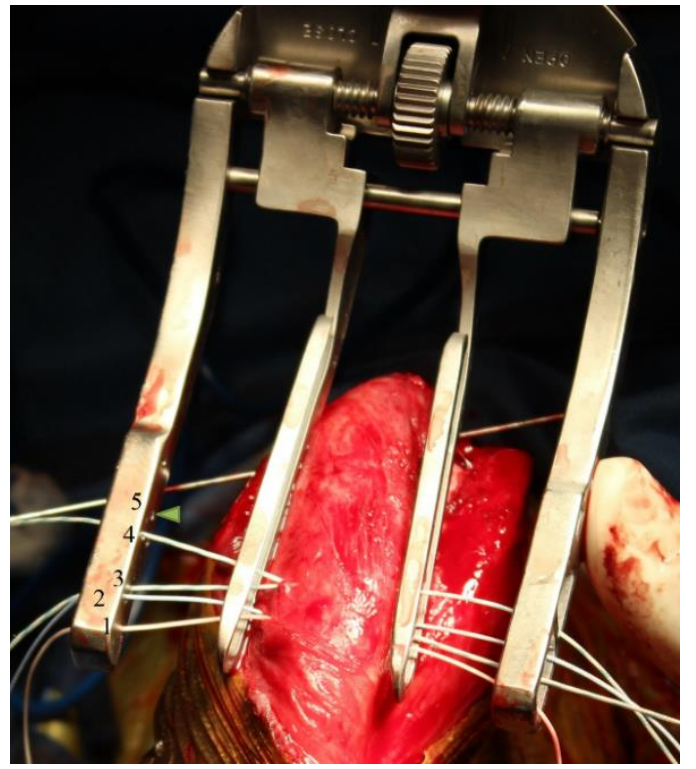

Fig. 3. Intraoperative photograph of FiberWire suture placement using the PARS Achilles Jig System. (1): transversely passed distal suture; (2): first transverse pass of self-locking middle suture; ( 3 and 4 ): obliquely passed special loop sutures used to pull the end of the middle suture through the tendon to create a self-locking loop; (5): empty hole (arrow head) for passage of the proximal transverse suture. 
A $1.5 \mathrm{~mm}$ drill bit was used to make three bone tunnels into the patella. Two axial bone tunnels were made to accept the three strands of FiberWire that had been placed through the patellar tendon for the primary tendon repair. A transverse medial-to-lateral bone tunnel was made to accommodate a patellotibial suture. The locations of the bone tunnels were maintained by placing $0.9 \mathrm{~mm}$ Kirschner wires within each of the bone tunnels (Figure 4).

The axial Kirschner wires were removed and the free ends of the three FiberWire sutures were threaded through each of the axial bone tunnels from distal to proximal, using a $0.8 \mathrm{~mm}$ suture passing wire with nitinol loop (Arthrex Inc, Naples, FL USA). The suture ends were then passed through a $3.5 \mathrm{~mm} 2$-hole oblong titanium button (Arthrex Inc, Naples, FL USA) seated within the quadriceps tendon at the apex of the patella. The paired ends of each FiberWire suture were tied individually over the button while the stifle was maintained in a normal standing angle.

A polyethylene ethyl ketone (PEEK) suture anchor system (Swivelock, Arthrex Inc, Naples, FL USA) was used to anchor the patellotibia suture to the tibial tuberosity. A $2.7 \mathrm{~mm}$ hole was drilled in cranial aspect of the proximal tibial tuberosity immediately distal to the insertion of the patellar tendon. This hole was tapped with a $3.5 \mathrm{~mm}$ Swivelock Tap (Arthrex Inc, Naples, FL USA).

The Kirschner wire in the transverse patellar bone tunnel was removed and a single strand $2 \mathrm{~mm}$ Fibertape (Arthrex Inc, Naples, FL USA) was threaded through the transverse patellar bone tunnel. The two free ends of the Fibertape were passed through the eyelet of a 3.5 $\mathrm{mm}$ Swivelock anchor. The anchor and suture were seated into the previously tapped hole in the proximal tibial tuberosity.

The joint capsule was closed routinely using \#0 polydioxanone suture (PDS II, Ethicon, Inc, Somerville, NJ, USA) in an interrupted cruciate pattern. Autologous Conditioned Plasma (Arthrex Inc, Naples, FL USA) (ACP, $3 \mathrm{~mL}$ ) was injected into the proximal patellar tendon after closure of the joint capsule. The fascial layer was closed with 2-0 polydioxanone in a simple continuous pattern. The subcutaneous and intradermal layers were then closed in a standard fashion using 3-0 mm poliglecaprone 25 (Monocryl, Ethicon, Inc, Somerville, NJ, USA) suture.

Immediate post-operative orthogonal radiographs confirmed normal positioning of the patella with the ratio of patellar tendon length to patella length measuring 2.01.

The bone tunnels and anchor location were all visible in the patella and tibial tuberosity, respectively. The oblong button implant was located proximal to the patella with likely soft tissue interposed between the button and the patella (Figure 5).

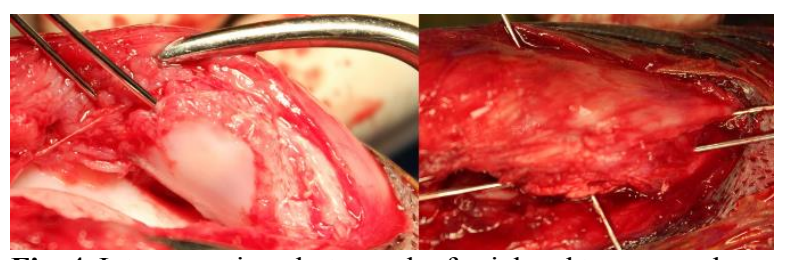

Fig. 4. Intraoperative photograph of axial and transverse bone tunnels with 0.035 " K-wires in place of tunnels (proximal is to the right).

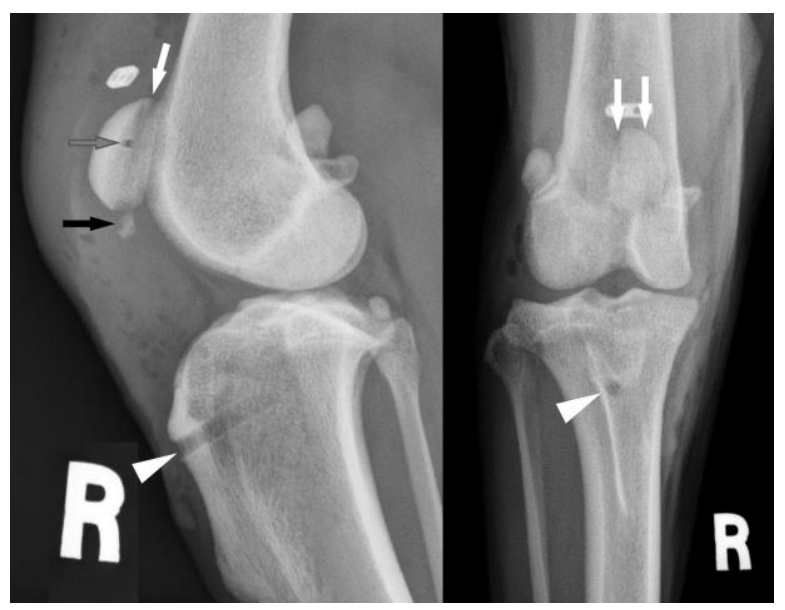

Fig. 5. Postop operative orthogonal radiographic images of the stifle. The patellar tendon remains markedly thickened. The avulsion fracture fragment is now closer to the patellar apex, but otherwise remains unchanged (black arrow). Axial bone tunnels are apparent in both views (white arrows). The transverse patellar tunnel can be seen on the lateral projection (gray arrow). The polyethyl ethyl ketone (PEEK) anchor location can be seen in the proximal tibial (arrow head).

Post-operatively the dog was administered carprofen, tramadol (Amneal Pharmaceuticals of NY, Hauppauge, NY, USA), cephalexin (Teva Parmaceuticals USA, Sellersville, PA, USA), and trazadone (Teva Parmaceuticals USA, Sellersville, PA, USA). The right pelvic limb was measured for a stifle orthotic device (K-9 Orthotics and Prosthetic, Beaver Bank, Nova Scotia, Canada) to limit post-operative range of motion before placing the limb in a lateral coaptation splint that extended from the inguinal region above the stifle to the digits.

\section{Post-operative care and outcome}

The dog was confined to a cage with instructions for exercise restriction for 8 weeks. The lateral splint was maintained for 3 weeks with weekly bandage changes until the custom, controlled range of motion orthosis arrived. Low-level laser therapy (LLLT) and lowintensity therapeutic ultrasound treatments were administered weekly for 4 weeks. An additional ACP injection $(3 \mathrm{~mL})$ was administered into the patellar tendon and periarticular tissues 3 weeks following surgery at which time the orthosis arrived. The dog was sent home with instructions to wear the orthosis at all 
times to allow limited range of motion of the stifle. Another ACP injection at 4 weeks following surgery and the controlled range of motion was increased from 0 to 30 degrees during weeks 4 through 7 . Low-level laser therapy (LLLT) and low-intensity therapeutic ultrasound treatments were administered at recheck examinations at weeks 6 and 7. Unfortunately, the orthosis caused several superficial abrasions after 3 weeks of constant use. These wounds were refractory to management while the orthosis was maintained. Therefore, a soft cast (3M Health Care, St. Paul, MN, USA) coaptation splint was used for stifle immobilization during the $8^{\text {th }}$ post-operative week, before discontinuing stifle immobilization.

The dog subjectively had a normal gait and posture without evidence of lameness when examined 3 months after surgery. Objective gait analysis (Gait4Dog, CIR Systems Inc, Franklin, NJ, USA) revealed mild hind limb asymmetry with reduced weight bearing noted in the right hind limb. Right hind Total Pressure Index (TPI) percentage of $18.1 \%$ compared to the left hind limb of $20.1 \%$, and a left to right hind pressure index ratio of 1.14 indicating decreased weight bearing in the right hind limb. The patellar tendon was moderately thickened on palpation; the swelling was notably decreased compared to initial presentation. The stifle had a full range of motion without a discernable pain response elicited on manipulation.

Radiographic examination of the right stifle revealed increased patellar tendon length compared to immediate post-operative radiographs, but comparative improvement to that of the pre-operative radiographs with a PTL:PL of 2.47 (Figure 6).

The location of the implants was unchanged. An ultrasound was performed that found the right patellar tendon fibers were arranged in a more organized fashion and were contiguous with the apex of the patella (Figure 7).

The dog was brought back to the clinic 3.6 years postoperatively for evaluation of an unrelated condition. The owner reported no concerns with the right stifle and that the dog had resumed normal activity with no known limitations. The right stifle was radiographed and the bone tunnels were still identifiable. The position of the button proximal to the patella was unchanged from that on previous radiographic images. The avulsed fragment off the distal pole of the patella had enlarged and the patella tendon was thickened, but the patella was positioned appropriately in the trochlear groove of the femur and there were no degenerative changes noted in the stifle (Figure 6).

\section{Discussion}

Surgical repair of a proximal patellar tendon avulsion in the dog reported here posed several challenges. There was no tendon tissue attached to the apex of the patella, which precluded us from performing a direct tenorrhaphy as described for mid-substance ruptures (Piermattei et al., 2006; Shipov et al., 2008; Kowaleski et al., 2012; Das et al., 2014). Previous reports have primarily focused on suture patterns to address midsubstance tendon ruptures in dogs (Piermattei et al., 2006; Shipov et al., 2008; Kowaleski et al., 2012; Das et al., 2014); however, the Bunnel-Mayer, the Kessler locking-loop, the three-loop pulley, and the Krackow suture patterns can be adapted to reattach tendon directly to bone (Moores et al., 2004; DeCamp et al., 2016). Previous biomechanical studies assessing gastrocnemius tendon repair found the double Krackow to be superior to the three loop pulley, and the three loop pulley to be superior to locking loop sutures when attaching tendon to bone (Moores et al., 2004; Wilson et al., 2014). We utilized FiberWire to maximize suture strength as we were very concerned about suture failure in light of the dog's weight, activity level, and the chronicity of the injury. Use of traditional monofilament nylon or polypropylene suture would have required a comparatively larger diameter suture than that of the FiberWire in order to achieve equivalent suture strength (Wüst et al., 2006). Use of larger diameter suture would have required larger diameter bone tunnels, increasing the potential for post-operative patellar fracture. FiberWire has nearly twice the tensile strength and a higher resistance to elongation compared to similar diameter monofilament sutures, thereby allowing for smaller bone tunnels (Burgess et al., 2010; Wilson et al., 2014). The use of FiberWire in a threeloop pulley pattern was considered, but there were concerns that proper tension and apposition of the tendon would be difficult to achieve due to this suture's substantial tissue drag, or that substantial parenchymal trauma would be induced when attempting to achieve proper tendon to bone apposition. Additionally, the modified three-loop pulley described for reattachment of a dog's tendon to bone requires suture that passes through the tendon at $60^{\circ}$ divergences with each pass of the suture (Moores et al., 2004); FiberWire used in this pattern could cause abrasions to the femoral articular cartilage as a minimum of two of the strands would theoretically be in direct contact with the trochlear groove. Intra-articular braided sutures have been shown to be abrasive when the material is in direct contact with the cartilage surface (Wüst et al., 2006). The technique employed in the dog reported here limited direct contact of the suture with the cartilage surface. The proprietary tendon repair jig system employed in this case utilizes three separate strands of sutures, each strand tied to itself, as opposed to one continuous suture and a single knot, had a modified three-loop pulley suture pattern been utilized. Increased security of the repair was hypothesized given that failure of one knot or strand would not necessarily cause failure of the entire repair. 

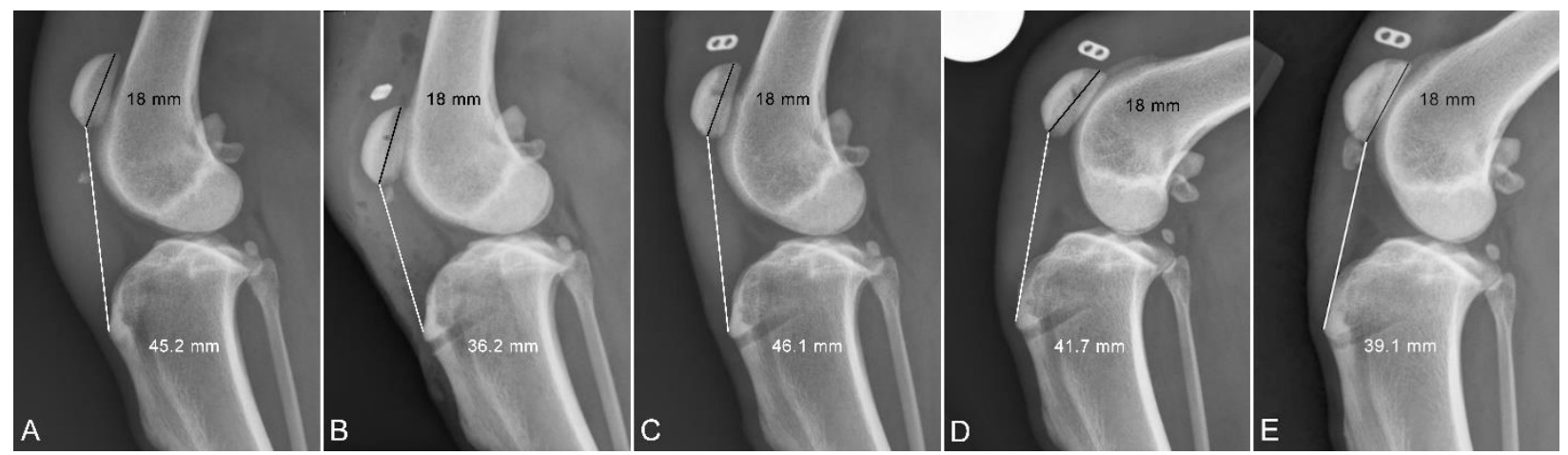

Fig. 6. Lateral projection radiographs of the stifle. (A): preoperative with a PLL:PL of 2.52; (B): post-operative ratio of 2.01; (C): 1 month post-operative, ratio of 2.55; (D): 2 month post-operative ratio of 2.31 Post-operative ratio increased moderately but maintained improved ratio compared to preoperative ratio; $(\mathbf{E}): 3.6 \mathrm{yr}$ post-operative, ratio of 2.17, bone tunnels are still evident.

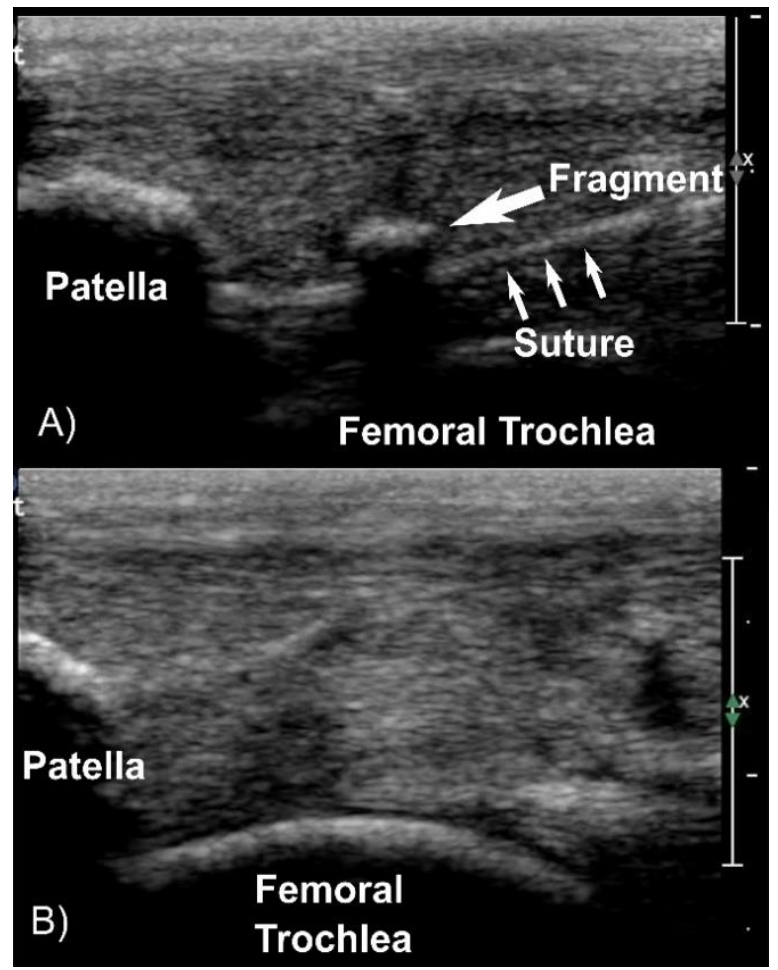

Fig. 7. (A): The FiberWire suture from the primary patellar tendon repair is seen distal to the patella. The irregular, welldefined hyperechoic structure causing distal acoustic shadowing distal to the patella is unchanged. (B): The fibers within the patellar tendon are arranged in a more organized fashion and the tendon margins are better defined, consistent with normal healing at the repair site.

Biomechanical testing would be required to compare the relative strength of these two techniques. The alternate FiberWire technique using the proprietary jig achieved our objective of directly opposing the patellar tendon to the apex of the patella in the dog reported here. An initial tenorrhaphy gap of $\leq 3 \mathrm{~mm}$ reportedly decreases the likelihood of repair failure (Gelberman $e t$ al., 1999), the avulsed end of the tendon was well opposed to the patella when the tenorrhaphy was completed.
In contrast to injury of the patellar tendon at its tibial insertion, the patella has limited bone available to accept suture anchors compared to the proximal tibia. Axially oriented trans-patellar bone tunnels were used to complete the primary repair. We theorized that the axial orientation would distribute the tensile forces more effectively than a single transverse bone tunnel, thereby minimizing the risk of iatrogenic patellar fracture.

Additionally, the axial orientation facilitated approximation of the end of the avulsed patellar tendon to the patella without interposition of suture material. We theorized that use of a titanium button would minimize trauma to the quadriceps tendon and mitigate subsequent suture laxity by limiting contact between the FiberWire suture and the peri-patellar soft tissues. Previous retrospective studies of patella tendon rupture advocate use of supplemental support techniques to protect the primary tendon repair (Shipov et al., 2008; Das et al., 2014). The first being placement of a patellato-tibia prosthesis (Smith et al., 2000; Shipov et al., 2008; Das et al., 2014).

The most commonly described techniques utilize monofilament nylon leader material placed through a bone tunnel in the tibial tuberosity followed by passage through a trans-patellar bone tunnel or passed circumferentially around the patella (Smith et al., 2000; Shipov et al., 2008; Das et al., 2014). Other authors have advocated use of stainless steel wire in a similar fashion (Vasseur, 2003; Piermattei et al., 2006; Kowaleski et al., 2012); wire utilized in this manner is prone to failure from cyclic fatigue when compared to monofilament nylon leader line (Das et al., 2014). A trans-patellar braided Fibertape suture was used for the patella-to-tibia support suture and was affixed to the tibial tuberosity via a knotless Swivelock anchor in the dog reported here.

The braided nature of the suture may provide increased strength and resist elongation or breakage better than nylon suture (Lawrence et al., 2005) and the Swivelock may increase repair security given the lack of a 
conventional knot and increased drag of the suture. Reattachment of tendon to bone takes considerable time to occur and remains weaker than normal control osseous tendon junction (Wang, 2006). The prolonged strength of the Fibertape compared to nylon in the support suture may have contributed to the success of the procedure.

We elected not using a taESF in this dog because we had concerns regarding potential morbidity associated with application of an external fixator to the proximal hind limb (Jackson and Pacchiana, 2004). We used a custom fabricated orthosis for stifle immobilization that allowed us to control maximal stifle flexion and extension angles. A gradual increase in stifle range of motion was hypothesized to facilitate better rehabilitation, to prevent quadriceps contracture (Carmichael and Marshall, 2012), to limit the degree of muscle atrophy and cartilage degeneration, and to promote more effective tendon healing (Henderson and Millis, 2014; Jaeger et al., 2005). Unfortunately, the orthosis caused multiple skin abrasions. Despite several modifications to the device, pressure sores persisted and use of the orthosis was discontinued 6 weeks following surgery. Thereafter a soft-cast splint was used to provide a passive reduction in range of motion. Future studies could evaluate the potential application of custom orthotics compared to taESF to determine the efficacy and morbidity associated with these modalities.

Patella alta is proximal displacement of the patella within the trochlear groove (Johnson et al., 2002, 2006; Mostafa et al., 2008). Several studies describe methods to measure proximodistal patellar alignment, with the most common calculation being the PLL:PL (Johnson et al., 2002, 2006; Mostafa et al., 2008). In this case, the pre-operative, immediate post-operative, 1 month, 2 month, and 3.6 year post-operative PLL:PL was 2.52, $2.01,2.55,2.31$ and 2.17 , respectively. The final PLL:PL measurement suggests that the patella remained in a mild patella alta position, as defined by Mostafa (Mostafa et al., 2008). Although the final 2.17 ratio is slightly greater than Mostafa's accepted 2.06 ratio, this may represent an individual variation in this dog, and is a substantial improvement from the preoperative PLL:PL. The patella tracked in a normal fashion and could not be luxated at the time of the longterm physcial examination.

The efficacy of the rehabilitative modalities used on the dog in this report to potentiate tendon healing are unclear. The use of ACP injections to promote tendon healing is controversial (Bosch et al., 2010; Georg et al., 2010; Estrada et al., 2014). Given the chronicity of the injury at the time of diagnosis, the authors considered it prudent to attempt to create a local environment around the injury that may promote healing. There are conflicting reports regarding the effects of isolated or combined application of therapeutic ultrasound and low-level laser therapy to enhance tendon healing (Enwemeka et al., 1990; Ng et al, 2003; Demir et al., 2004; Ng and Fung, 2007; Oliveira et al., 2009). Moreover, most studies evaluating low level laser light therapy and therapeutic ultrasound advocate for daily treatments as opposed to the intermittent treatments performed in this case. The authors unfortunately cannot ascertain whether these therapeutic modalities contributed to a successful outcome in the dog reported here.

This report describes a successful treatment of a proximal patellar tendon avulsion injury using a proprietary tendon repair system. The PARS allowed for precise placement of the suture strands within the tendon parenchyma. The strength and stiffness of the FiberWire and Fibertape contributed to a successful clinical outcome. Unfortunately this report has a number of limitations and direct comparisons cannot be made to previously reported techniques with regards to relative success, risks, or complications (Smith et al., 2000; Jaeger et al., 2005; Piermattei et al., 2006; Shipov et al., 2008; Kowaleski et al., 2012; Das et al., 2014). Further studies are warranted to directly compare the biomechanics of this repair technique to conventional techniques, and to elucidate differences in morbidity and repair failure in a larger group of randomized dogs. Such prospective clinical studies are limited by the comparative rarity of this naturally occurring injury in dogs.

\section{Conflict of interest}

The Authors declare that there is no conflict of interest.

\section{References}

Bosch, G., van Schie, H., de Groot, M., Cadby, J., van de Lest, C., Barneveld, A. and van Weeren, P.R. 2010. Effects of platelet-rich plasma on the quality of repair of mechanically induced core lesions in equine superficial digital flexor tendons: A placebocontrolled experimental study. J. Orthop. Res. 28(2), 211-217.

Burgess, R., Elder, S., McLaughlin, R. and Constable, P. 2010. In vitro biomechanical evaluation and comparison of fiberwire, fibertape, orthofiber, and nylon leader line for potential use during extraarticular stabilization of canine cruciate deficient stifles. Vet. Surg. 39(2), 208-215.

Cabassu, J., Ivanoff, S., Haroutunian, G. and Besse, J. 2001. Rupture bilatérale des ligaments patellaires chez un chien pendant un traitement à l'enrofloxacine. Traitement. Revue Méd. Vét. 152(7), 523-530.

Carmichael, S. and Marshall, W. 2012. Muscle and tendon disorders. In: Tobias, K., Johnston, S., ed. Veterinary Surgery: Small Animal. $1^{\text {st }}$ ed. St. Louis, MO: Saunders/Elsevier, pp: 1127-1134. 
Das, S., Thorne, R., Lorenz, N., Clarke, S., Madden, M., Langley-Hobbs, S., Perry, K.L., Burton, N.J., Moores, A.L. and Mosley, J.R. 2014. Patellar ligament rupture in the dog: repair methods and patient outcomes in 43 cases. Vet. Rec. 175(15), 370.

DeCamp, C., Johnston, S.A., Dejardin, L.M. and Schaefer, S.L. 2016. Principles of joint surgery. In: Brinker, Piermattei and Flo's Handbook of Small Animal Orthopedics and Fracture Repair. $5^{\text {th }}$ ed. Saint Louis, MO: Saunders Elsevier, pp: 213-224.

Demir, H., Menku, P., Kirnap, M., Calis, M. and Ikizceli, I. 2004. Comparison of the effects of laser, ultrasound, and combined laser + ultrasound treatments in experimental tendon healing. Lasers Surg. Med. 35(1), 84-89.

Enwemeka, C., Rodriguez, O. and Mendosa, S. 1990. The biomechanical effects of low-intensity ultrasound on healing tendons. Ultrasound Med. Biol. 16(8), 801-807.

Estrada, R., van Weeren, R., van de Lest, C., Boere, J., Nagaly, R., Ionita, J.-C., Estrada, M. and Lischer, C. 2014. Effects of autologous conditioned plasma (ACP) on the healing of surgically induced core lesions in equine superficial digital flexor tendon. Pferdeheilkunde 30(6), 633-642.

Gelberman, R., Boyer, M., Brodt, M., Winters, S. and Silva, M. 1999. The effect of gap formation at the repair site on the strength and excursion of intrasynovial flexor tendons. J. Bone Joint Surg Am. 81(7), 975-982.

Georg, R., Maria, C., Gisela, A. and Bianca, C. 2010. Autologous conditioned plasma as therapy of tendon and ligament lesions in seven horses. J. Vet. Sci. 11(2), 173-175.

Henderson, A. and Millis, D. 2014. Response of musculoskeletal tissues to disuse and remobilization. In: Millis, D., Levine, D., ed. by. Canine Rehabilitation and Physical Therapy. $2^{\text {nd }}$ ed. St. Louis, MO: Saunders, pp: 92-153.

Hermanson, J. 2012. The muscular system: cranial muscles of the thigh. In: Evans, H., de Lahunta, A., ed. by. Miller's Anatomy of the Dog. $4^{\text {th }}$ ed. St. Louis, MO: Saunders, pp: 262.

Jackson, L.C. and Pacchiana, P.D. 2004. Common complications of fracture repair. Clin. Tech. Small Anim. Pract. 19(3), 168-179.

Jaeger, G., Wosar, M., Marcellin-Little, D. and Lascelles, B. 2005. Use of hinged transarticular external fixation for adjunctive joint stabilization in dogs and cats: 14 cases (1999-2003). J. Am. Vet. Med. Assoc. 227(4), 586-591.

Johnson, A., Broaddus, K., Hauptman, J., Marsh, S., Monsere, J. and Sepulveda, G. 2006. Vertical patellar position in large-breed dogs with clinically normal stifles and large-breed dogs with medial patellar luxation. Vet. Surg. 35(1), 78-81.

Johnson, A., Probst, C., DeCamp, C., Rosenstein, D., Hauptman, J. and Kern, T. 2002. Vertical position of the patella in the stifle joint of clinically normal large-breed dogs. Am. J. Vet. Res. 63(1), 42-46.

Johnson, K.A. 2014. Piermattei's atlas of surgical approaches to the bones and joints of the dog and cat. $5^{\text {th }}$ ed. Philadelphia, Pa. Saunders, pp: 396-400.

Kowaleski, M., Boudrieau, R. and Pozzi, A. 2012. Stifle joint. In: Tobias, K., Johnston, S., ed. by. Veterinary Surgery: Small Animal. $1^{\text {st }}$ ed. St. Louis, MO: Elsevier Saunders, pp: 991-992.

Lawrence, T., Woodruff, M., Aladin, A. and Davis, T. 2005. An assessment of the tensile properties and technical difficulties of two- and four-strand flexor tendon repairs. J. Hand Surg. Br. 30(3), 294-297.

Montgomery, R. and Fitch, R. 2015. Muscle and tendon disorders. In: Slatter, D., ed. by. Textbook of Small Animal Surgery. $3^{\text {rd }}$ ed. Philadelphia, PA: Saunders, pp: 2264-2272.

Moores, A., Comerford, E., Tarlton, J. and Owen, M. 2004. Biomechanical and clinical evaluation of a modified 3-loop pulley suture pattern for reattachment of canine tendons to bone. Vet. Surg. 33(4), 391-397.

Mostafa, A., Griffon, D., Thomas, M. and Constable, P. 2008. Proximodistal alignment of the canine patella: radiographic evaluation and association with medial and lateral patellar luxation. Vet. Surg. 37(3), 201-211.

Ng, C., Ng, G., See, E. and Leung, M. 2003. Therapeutic ultrasound improves strength of Achilles tendon repair in rats. Ultrasound Med. Biol. 29(10), 1501-1506.

Ng, G. and Fung, D. 2007. The effect of therapeutic ultrasound intensity on the ultrastructural morphology of tendon repair. Ultrasound Med. Biol. 33(11), 1750-1754.

Oliveira, F., Pinfildi, C., Parizoto, N., Liebano, R., Bossini, P., Garcia, É. and Ferreira, L.M. 2009. Effect of low level laser therapy $(830 \mathrm{~nm})$ with different therapy regimes on the process of tissue repair in partial lesion calcaneous tendon. Lasers Surg. Med. 41(4), 271-276.

Piermattei, D.L., Flo, G.L. and DeCamp, C. 2006. The stifle joint. In: Brinker, Piermattei and Flo's Handbook of Small Animal Orthopedics and Fracture Repair. $4^{\text {th }}$ ed. Saint Louis, MO: Saunders Elsevier, pp: 562-632.

Shipov, A., Shahar, R., Joseph, R. and Milgram, J. 2008. Successful management of bilateral patellar tendon rupture in a dog. Vet. Comp. Orthop. Traumatol. 21(2), 181-184.

Smith, M., de Haan, J., Peck, J. and Madden, S. 2000. 
Augmented primary repair of patellar ligament rupture in three dogs. Vet. Comp. Orthop. Traumatol. 13(3), 154-157.

Vasseur, P. 2003. Stifle joint. In: Slatter, D., ed. by. Textbook of Small Animal Surgery. $3^{\text {rd }}$ ed. Philadelphia, PA: Saunders, pp: 2128.

Wang, J.H.C. 2006. Mechanobiology of tendon. J. Biomech. 39, 1563-1582.

Wilson, L., Banks, T.A., Luckman, P. and Smith, B.
2014. Biomechanical evaluation of double Krackow sutures versus the three-loop pulley suture in a canine gastrocnemius tendon avulsion model. Aust. Vet. J. 92, 427-432.

Wüst, D., Meyer, D., Favre, P. and Gerber, C. 2006. Mechanical and handling properties of braided polyblend polyethylene sutures in comparison to braided polyester and monofilament polydioxanone sutures. Arthroscopy 22(11), 1146-1153. 